\title{
A NEW PULSED-MODE MICROMACHINED FLOW SENSOR FOR AN INTEGRATED MICROFLUIDIC SYSTEM
}

\author{
Nihat Okulan, H. Thurman Henderson and Chong H. Ahn \\ Center for Microelectronic Sensors and MEMS \\ University of Cincinnati \\ Department of Electrical and Computer Engineering \\ P.O. Box 210030, Cincinnati, Ohio 45221-0030
}

\begin{abstract}
A new pulsed-mode micromachined flow sensor for an integrated microfluidic system has been designed, fabricated and characterized. This micro flow sensor has several unique physical and operational characteristics, as a part of the complex of components constituting a generic fully integrated microfluidic system $[1,2]$ for autonomous bio-chemical analysis. Companion elements include microvalves, micropumps, microreservoirs, microchannels, etc., whose liquid (or gaseous) fluid dynamics must be characterized by these micromachined sensors.
\end{abstract}

\section{Introduction}

In contrast to other thermally-based flow sensors [3], here the sensing elements are located in the microchannel walls. This novel design prevents clogging by virtue of dead volume or other interference which may cause nucleation of particle deposition. Nevertheless, the single crystalline silicon elements are thermally isolated from the bulk by unique low stress, low temperature-deposited $\left(<150{ }^{\circ} \mathrm{C}\right) \mathrm{PECVD} \mathrm{SiO}_{2}$ [4], which is considerably superior to other electrical and thermal isolation schemes. In essence, the sensor measures liquid flow through the integrated channel sidewall.

In order to minimize possible thermally induced effects in the fluid, the elements are pulsed at a fixed current level (typically $2.5 \mathrm{~mA}$ ) for 50 to $100 \mathrm{~ms}$ intervals at a low duty cycle, typically $2-4$ per second. High resistivity $(50 \mathrm{Ohm}-\mathrm{cm})$ silicon results in a highly sensitive element, which easily achieves full operation during the on-cycle, because of the fast time response resulting from miniature size and thermal isolation.

Unamplified sensitivities up to $25 \mathrm{mV}$ at $10 \mathrm{ul} / \mathrm{min}$ are achieved. However, a novel negative differential resistance (NDR) modality is also possible by (stable) transient operation in thermal run-away. Depending on the several modalities made possible, the sensitivity can be enhanced by taking advantage of the onset of intrinsic operation or by deep impurity operation (not covered here in detail).

In contrast to earlier in-flow immersion, the electrodes are located outside the channel and thus do not need further electrical passivation. The thermal field can be made to variously penetrate the boundary layer appropriately. A numerical simulation of the liquid flow field [6] and thermal distribution has been realized using ANSYS 53 and the FLOTRAM CFD option.

Other members of our research team have also developed effective electronics for integrating the digital sampling to achieve high resolution measurement of total flow volume, however this will be reported separately elsewhere.

\section{Design and Fabrication}

Since thermal devices are dependent on differential temperatures, it was essential to make use of an effective thermal isolation structure. Figure 1 shows an SEM micrograph of the top view of the fabricated flow sensor. Thermal isolation was achieved by constructing a physical isolation channel between the

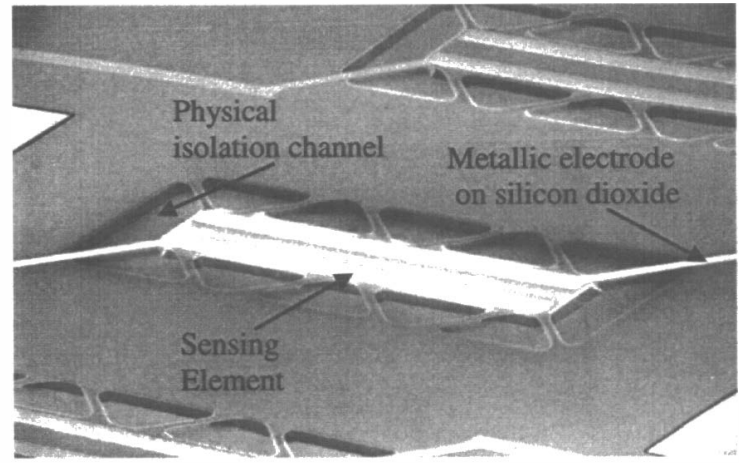

Figure 1. SEM micrograph of the top of a linear array of three fabricated flow sensors

sensing element and the surrounding bulk silicon using the low stress silicon dioxide bridges [4], which are approximately an order of magnitude better than silicon nitride for thermal isolation. Electrically conducting layers from the bulk to the sensing element where formed over two of the bridges in order to access the electrodes on the sensing element. The physical channel was generated by aligning the edges of windows to the $\{111\}$ crystallographic planes of the underlying (100) silicon, opening these windows in the $\mathrm{SiO}_{2}$ and etching away the silicon during a $\mathrm{KOH}$ anisotropic etching process [3].

Compared to other thermally based flow sensors a significant advantage of this micro flow sensor is its detailed design. Complex electrical passivation procedures are not necessary since the sensing elements are integrated in the microchannel walls. As can be seen in Fig.2, the electrodes are located outside the flow channel. Furthermore, having the sensing elements not immersed in the flow

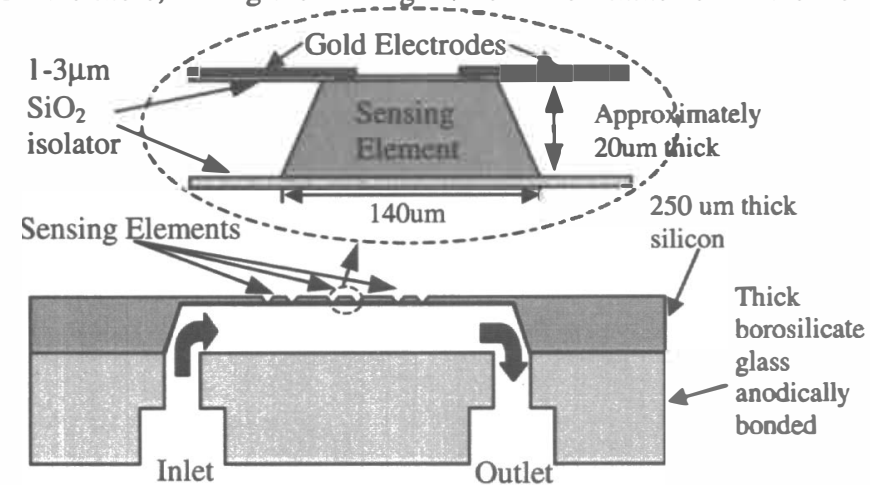

Figure 2: Physical structure of the three-sensor array. The array allows the alternative method of upstream and downstream temperature measurement.

stream prevents possible clogging and dead spaces. The shape of the etched channel allows a convenient packaging scheme for a multi- 
level bonded package in an integrated biochemical "lab-on-achip" [2]. These very novel arrangements specifically add reliability and simplicity for this specific application.

\section{Operating Principle}

The sensing element is pulsed at a fixed current level in time intervals of 50 to $100 \mathrm{~ms}$ at a relatively low duty cycle at 2 4 times per second. This measuring scheme results in high sensitivity and repeatability. At the same time this scheme is simple and economical; and it allows a simple driving circuitry, consequently saving chip space and battery lifetime.

Choosing the appropriate duty cycle is of importance, since a high duty cycle could lead to an elevated fluid temperature that could alter the properties of the biochemistry being performed. On the other hand, a to low duty cycle could degrade the accuracy (e.g. an unacceptable low sample rate).

During the operational mode, the sensor is typically pulsed with $2.5 \mathrm{~mA}$ for $100 \mathrm{~ms}$ with a duty cycle of $25 \%$. The temperature of the sensing element, $T_{s}$, will be elevated to a value above the fluid temperature, $T_{f}$, depending on the flow rate of the fluid. The increase in $T_{s}$ leads to an increase in sensing element resistance and thus to an increase in voltage drop across the resistor.

The behavior of the voltage drop across the sensing element due to electrical current flow during the pulsing period is shown in Fig. 3. The output voltage is plotted as a function of time for various flow rates. The output voltage increases in a

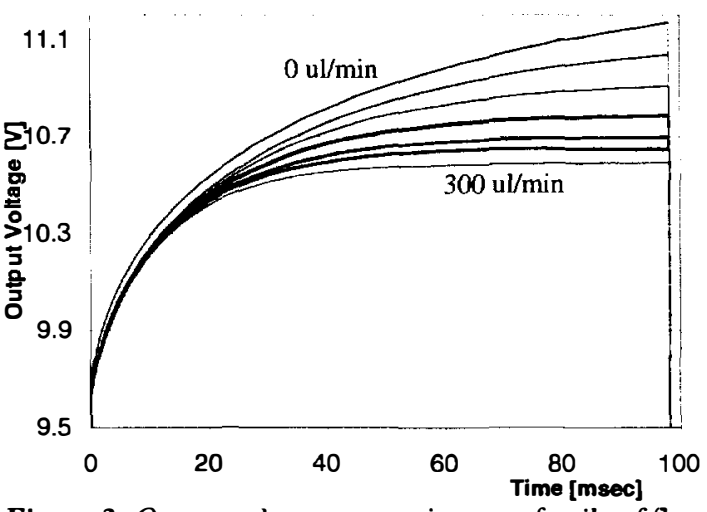

Figure 3: Output voltage versus time at a family of flow rates from 0 to $300 \mathrm{ul} / \mathrm{min}$ separated in $50 \mathrm{ul} / \mathrm{min}$ steps

saturation curve with a time constant $\tau=R_{t h} C_{t h}$, where $R_{t h}$ is the system thermal resistance and $C_{t h}$ the thermal capacitance, related to sensor size.

When there is no flow, the power dissipated by the sensing element causes a conductive heat flow into the fluid and the surrounding bulk silicon. If liquid passes, a forced convective heat flow is added, which drops the temperature of the sensing element and decreases the thermal resistance. The drop of temperature leads to a decreased electrical resistance of the sensing element and thus to a drop in output voltage. In addition, the decrease in thermal resistance leads to a smaller $\tau$ because of the added (shorting) thermal conductance. The output voltage or the time constant can alternatively be used as an indicator for the flow rate.

To determine the fluid temperature for on-line temperature correction to a changing ambient, the resistance of the sensing element was measured by using a very low current pulse of 100uA just before pulsing the sensor. The low current ambient temperature-measuring pulse avoids any significant heating and thus does not interfere with the flow rate measurement.

The basic operation is not unlike the classical "hot-wire anemometer." To understand the relationship between sensing element resistance and temperature, and to understand the relationship between the sensing element temperature and the flow rate, a brief theory is presented.

\subsection{Sensing Element Temperature Versus Flow Rate}

The heat flux from the sensing element increases with the flow rate. In addition to the heat conveyed away by the flow, $Q_{\text {conv }}$ (forced heat convection), heat is conducted, $Q_{\text {cond, }}$ to the fluid and carrier substrate in which the sensor is imbedded. The radiated heat is negligible. In steady state, the heat transfer is balanced by the power dissipation $P$ in the sensor:

$$
P=Q_{\text {cond }}+Q_{\text {conv }}
$$

The flow measurement depends on the relationship between $Q_{\text {conv }}$ and the flow (other secondary heat transfer effects, such as radiation and natural convection, can be included in $\left.Q_{\text {cond }}\right)$. The amount of heat $Q_{\text {conv }}$ transferred to the fluid is proportional to the temperature difference between the hot sensing element, $T_{s}$, and the fluid temperature, $T_{f}$ :

$$
Q_{c o n v}=G_{c o n v}\left(T_{s}-T_{f}\right)
$$

where $G_{c o n v}$ is the heat conductance due to forced convection and is a function of the flow velocity.

Considering laminar flow and the decreasing thermal boundary layer with increasing velocity, the heat transfer increases in relation with the flow velocity $U$. The surface heat flux per unit area $Q_{\text {conv }}$ is given by

$$
Q_{c o n v}^{\prime}=0.332 k_{f}\left(T_{s}-T_{f}\right)\left(\sqrt[3]{P_{r}}\right) \sqrt{U /(x v)}
$$

where $k_{f}$ and $v$ are the thermal conductivity and kinematic viscosity, respectively, of the fluid and $P_{r}$ is the Prandtl number; position $x$ is measured from the upstream edge of the sensing element.

Integrating $Q_{\text {conv }}^{\prime}$ over the entire surface (which is determined by the length and width of the sensing element) and dividing by $\left(T_{s}-T_{f}\right)$ results in $G_{\text {conv }}$. Thus, $G_{\text {conf }}$ increases in proportion to $U^{1 / 2}$ for laminar flow. The relation between power dissipation, the flow velocity and the temperature difference between $T_{s}$ and $T_{f}$ can be written as

$$
P /\left(T_{s}-T_{f}\right)=G_{0}+K \sqrt{U}
$$

where $K$ is the flow sensitivity (a geometrical term), and the zeroflow thermal conductance is $G_{o}$. These are parameters which for a simple sensor geometry can be estimated from theoretical analysis. Solving equation (4) for the sensing element temperature results in:

$$
T_{s}=T_{f}+P /\left(G_{0}+K \sqrt{U}\right)
$$

Figure 4 illustrates the relationship between sensing element temperature and flow velocity.

\subsection{Sensing Element Resistivity Versus Temperature}

Since the sensitivity of the micro flow sensor depends primarily on the change in electrical resistivity with temperature, it is important to review the relevant classical device physics of the micro flow sensor. The relationship for electrical resistivity $\rho$ is a function of temperature $T$ and is given as

$$
\rho^{-1}(T)=q\left[n(T) \mu_{n}(T)+p(T) \mu_{p}(T)\right]
$$


where $q$ is the magnitude of electronic charge, $n_{(T)}$ and $p_{(T)}$ are free electron and hole concentrations, respectively, and $\mu_{n(T)}$ and $\mu_{p(T)}$ are electron and hole mobilities, respectively. The

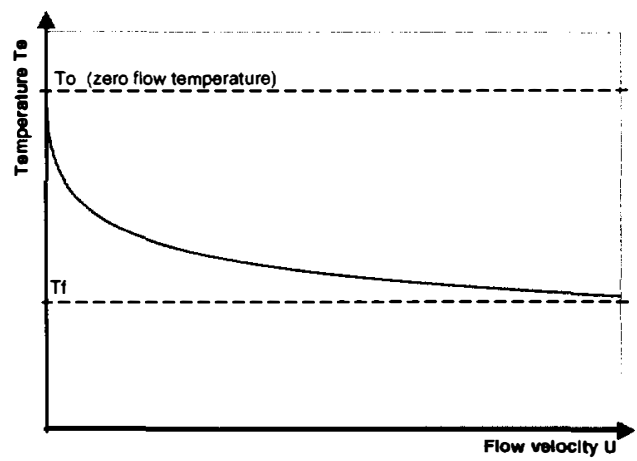

Figure 4: Element temperature versus velocity (proportional to flow rate)

temperature dependence of the mobility in the range of interest is approximately proportional to $T^{2 / 3}$ (a more detailed relationship was used in the calculation). To determine the free carrier concentrations the charge neutrality equation must be solved. The micro flow sensors where fabricated using $50 \mathrm{Ohm}$ $\mathrm{cm}$, n-type silicon, which corresponds to a phosphorus concentration, $N_{d}$, of $8.5 \mathrm{E} 13 \mathrm{~cm}^{-3}$. Hence, the charge neutrality equation reduces to

$$
n=p+N_{d}
$$

and

$$
\begin{aligned}
& p\left(T, E_{f}\right)=N_{v} \exp \left[\frac{-\left(E_{f}-E_{v}\right)}{k T}\right] \\
& n\left(T, E_{f}\right)=N_{c}(T) \exp \left[\frac{-\left(E_{c}-E_{f}\right)}{k T}\right]
\end{aligned}
$$

where $N_{c}(T)$ and $N_{v}(T)$ are conduction and valence band effective densities of states, $E_{c}$ and $E_{v}$ are conduction and valence band edges, $k$ is the Boltzman constant and $E_{f}$ is the Fermi level.

Solving the charge neutrality equation transcendentally for $E_{f}$, and using $E_{f}$ to solve for the electron and hole concentration, the resistivity at any temperature $T$ can be calculated. Figure 5 shows the calculated resistivity over the range from $0-300{ }^{\circ} \mathrm{C}$.

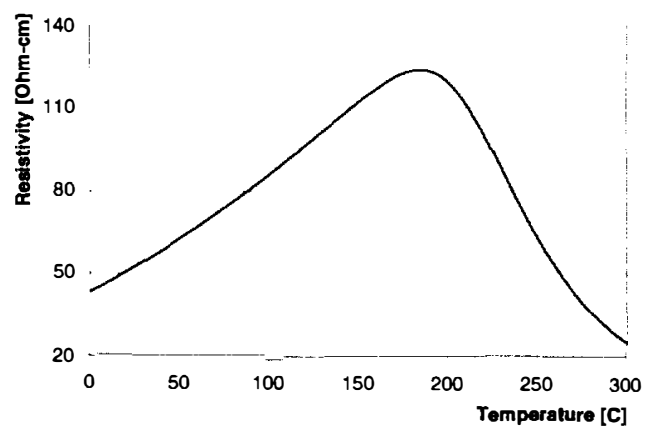

Figure 5: Resistivity versus temperature

The current is constant during each heating pulse, and the heating is thus proportional to the resistivity, resistance or voltage. Thus, it can be seen that the sensor can be operated in two modalities. In the lower temperature ranges, one can take advantage of the positive differential resistance (PDR) modality.
However, a novel negative differential (NDR) modality is also possible by (stable) transient operation in "thermal run-away". This would lead to an increase in sensitivity but as a trade-off would have to be operated in higher temperature ranges. An alternative modality, not covered here but to be later published, is to achieve an even higher sensitivity using a deep impurity such as gold, with a relatively low operating temperature.

\section{Finite Element Analysis}

The numerical simulation of the liquid flow field and thermal distribution has been realized using ANSYS 53 and the FLOTRAN CFD option. The CFD program solves the coupled fields of temperature and flow. A simple two-dimensional model was calculated where the channel was reduced to a rectangular configuration with the dimensions of the real sensor. Three sensing elements were located in the middle of the flow field and a constant heat source was applied to the sensing element in the center. (Of course an alternative flow measurement scheme [5] is to use the center element merely as a heater then measure flow by virtue of the incremental resistance of the up and downstream sensor.)

The liquid flow was measured by its influence on the temperature distribution in the channel resulting from the heat generated from the center element. The analysis showed that the temperature change of the center sensing element with respect to the flow rate was much larger than the upstream and downstream located sensing elements. Figure 6 indicates the temperature of the center sensing element as a function of flow rate.

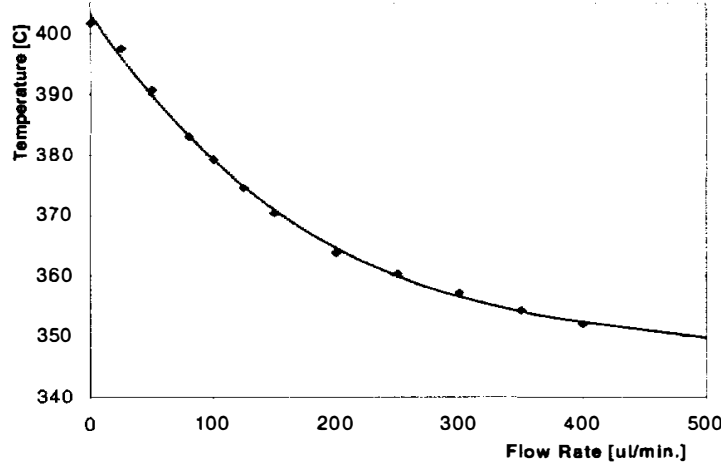

Figure 6: Sensing element temperature versus flow rate

As expected, the temperature consistently decreases with the flow. As a conclusion, one achieves more sensitivity using only a singular "heating-sensing" element, which can also be used to sample ambient temperature, as noted earlier.

However, at these MEMS dimensions such traditional modeling tools have limited validity but can still be used for proof of concept.

\section{Experimental Results and Discussion}

The measuring system consists of a LC-pump (which will be replaced with an integrated pump in the eventual microfluidic system) with adjustable flowrates from $10 \mathrm{ul} / \mathrm{min}$ to $10 \mathrm{ml} / \mathrm{min}$, a data acquisition module that can record data up to $100 \mathrm{~K}$ samples/s with a 12 bit resolution, a voltage controllable current source and a control computer. It allowed automatic measuring and recording of experimental results. Water was used for testing the sensor.

Figure 8 shows that the measured temperature dependence of the sensing element resistance agrees rather well with the calculated theoretical temperature dependence. The resistance was calculated by multiplying the above determined resistivity with the dimensional factor of the sensor.

Measurements of the flow rates were performed by arbitrarily adjusting a specific flow rate at the pump and gathering data during 


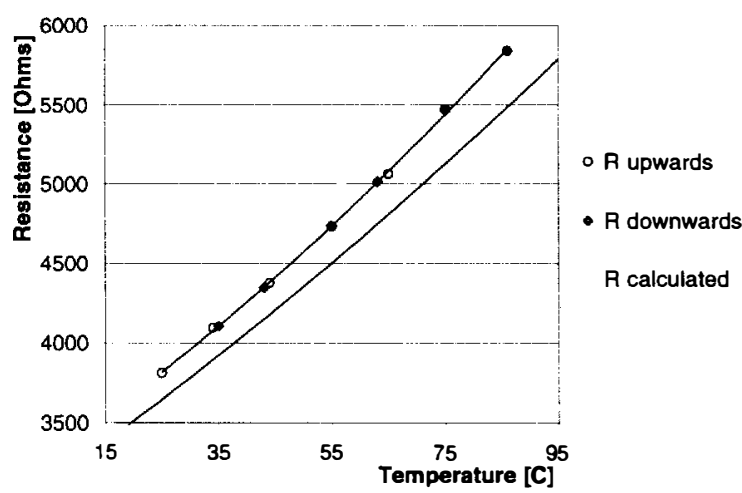

Figure 8: Resistance versus temperature

50 duty cycles. For simplicity, an average value of the last 20milliseconds (1500 measurements) of each output voltage pulse was evaluated and used as an indicator for the flow rate. Figure 9 shows the output voltage versus flow rate. The output

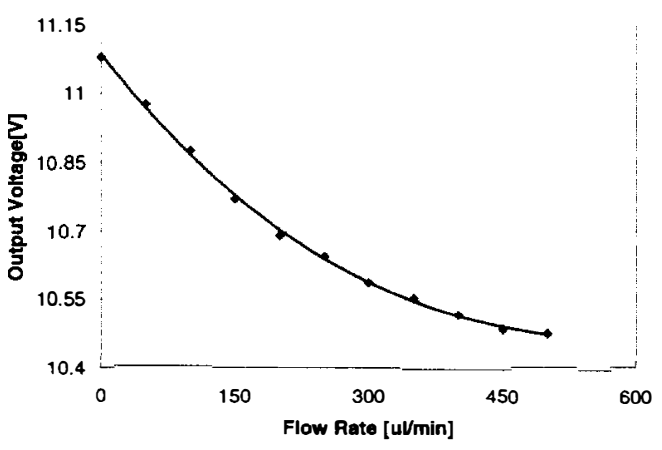

Figure 9: Output voltage versus flow rate

voltage drops with increasing flow rates, because of cooling, and has the experimental shape as was analytically derived. Unamplified sensitivities up to $25 \mathrm{mV}$ per $10 \mathrm{ul} / \mathrm{min}$ were achieved.

Flow measurements for three selected fluid temperatures were taken. The output voltage as a function of fluid temperature and flow rate is shown in Fig. 10. As one can see,

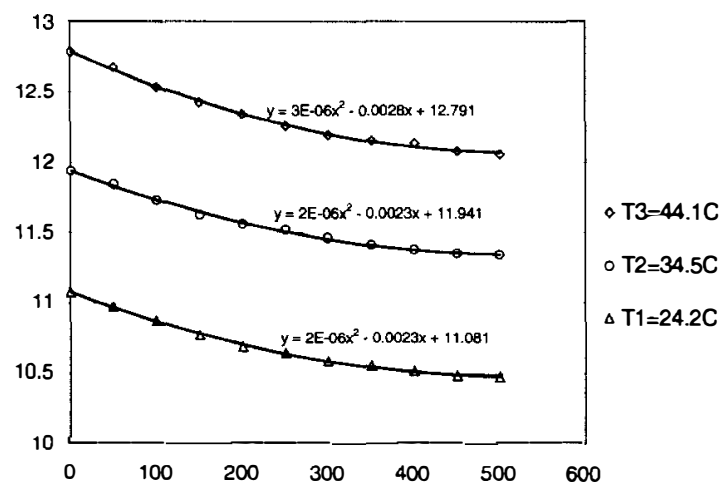

Figure 10: Output voltage versus temperature and flow rate

the behavior of the output voltage versus flow rate can be described with similar curve fitted relationships where each fit begins at a different zero flow rate offset voltage $V_{0}$. The offset voltage is tied to the fluid temperature. As the fluid temperature increases, $V_{0}$ increases. Temperature compensation circuitry is being designed to allow a high degree of accuracy in the full range of operational temperatures for the associated generic microfluidic system.

\section{Conclusions}

A micro flow-sensor, which has also been rendered in smaller versions than herein reported, has been fabricated and dedicated to the measurement of small liquid flows for autonomous bio-chemical analysis. Due to well defined arrangements of the sensing elements integrated into the microchannel walls, clogging and dead spaces are minimized. Further, the complex electrical passivation of electrodes is eliminated. Thermal insulation is achieved by implementing a physical isolation channel between sensing elements and the bulk silicon, leading to a fast time response due to the small mass of the elements. Experimental data show promising results with fast and highly sensitive signals for regulation of microflow in the range of $0.001-10 \mathrm{ml} / \mathrm{min}$.

A pulsing modality minimizes thermally induced effects in the fluid and also allows minimization of potential drift. Further, this mode of operation saves valuable battery lifetime.

\section{Acknowledgements}

This research has been funded in part by DARPA contract P9619-80 under the Microflumes/ETO program directed by Dr. Rose Ritts, for use in a generic integrated microfluidic system. We acknowledge the associated flow sensor contributions of colleagues Gerd Berger and Karsten Klein. Also we wish to thank Dr. Thomas Mantei and his group, including F. Li and C.-T. Lin, for development of the special technique for deposition of the low stress silicon dioxide.

\section{References}

1. M. Elwenspoek, "Toward integrating microliquid handling system", J. Micromech. Microeng., 1994, Vol.4, p.227.

2. DARPA BBA96-19 No. P9619-80, "Generic microfluidic system for remote sensors", Unversity of Cincinnati, 1997.

3. Timothy M. Betzner, "Structural design and characteristics of a thermal isolated, sensitivity-enhanced, bulk-michromachined, silicon flow sensor", J. Micromech. Microeng., 1996, Vol.6, p.217.

4. J.Pilchowski, F. Li, C.-T. Lin, T. D. Mantei, H. T. Henderson, "Silicon dioxide deposition in high density plasma for packaging application", Proc. of The Electrochemical Society, $193^{\text {rd }}$ meeting, San Diego, Calif., May 3-8, 1998.

5. R.G.Johnson, R.E. Higashi, "A highly sensitive silicon chip microtransducer for air flow and differential pressure sensing applications", Sens. Actuators 11, 1987, p.63.

6. R. Zengerle, M. Richter, "Simulation of microfluidic systems", J. Micromech. Microeng., 1994, Vol.4, p.192 